\title{
Occurrence of autoantibodies for gastrointestinal autoimmune diseases in children with common variable immune deficiency and selected IgA deficiency
}

\author{
Anna Pituch-Noworolska, Anita Błaut-Szlósarczyk, Katarzyna Zwonarz \\ Department of Clinical Immunology, Polish-American Institute of Pediatrics, Jagiellonian University Medical College, Krakow, \\ Poland
}

Prz Gastroenterol 2013; 8 (6): 370-376

DOI: $10.5114 /$ pg.2013.39920

Key words: autoimmunity, common variable immune deficiency, IgA deficiency, immunoglobulin substitution, children.

Address for correspondence: Anna Pituch-Noworolska MD, PhD, Department of Clinical Immunology, Polish-American Institute of Pediatrics, Jagiellonian University Medical College, 265 Wielicka St, 30-663 Krakow, Poland, phone: +48 694 619 707, e-mail: mipituch@cyf-kr.edu.pl

\begin{abstract}
Introduction: Selected IgA deficiency (IgAD) and common variable immune deficiency (CVID) are humoral immunity deficiencies frequent in children. In both these types of immunodeficiency, autoimmune diseases are present in 20-30\% of patients, but the disease profiles are different between adults and children. Autoimmune diseases of the gastrointestinal tract (IBD) and celiac disease are typical for children with IgAD and CVID. Diagnosis is based on clinical symptoms, histology of jejunum and antibodies often preceding the onset of disease. However, the diagnosis of IBD and celiac disease is difficult in immune deficiency patients due to weaker or absent production of antibodies, and different jejunum histology, particular in CVID patients.

Aim: Detection of antibodies for autoimmune diseases in children with diagnosis of CVID and IgAD.

Material and methods: The study included 43 children with CVID and 63 children with IgAD diagnosis. Antibodies typical for celiac disease (for endomysium, tissue transglutaminase and gliadin) were tested in IgA class (CVID patients), IgG class (IgAD, CVID patients) and found in 16 patients (3-CVID, 13 - IgAD).

Results: Antibodies for IBD (for Saccharomyces cerevisiae antigen - ASCA, goblet cells - Gab, neutrophil's cytoplasm - ANCA, pancreatic cells - Pab) were noted in 17 patients (7-CVID, 10 - IgAD). Celiac disease was diagnosed in two children with mild and unspecific clinical symptoms followed by introduction of a gluten-free diet. The remaining children with present antibodies but without clinical symptoms involving the gastrointestinal tract are under careful clinical observation with antibody assay every 6 months.
\end{abstract}

Conclusions: The antibodies are produced despite impaired humoral immunity but the level might be low so the lower limit of positive results is postulated.

\section{Introduction}

The association between autoimmunity and humoral immune deficiency is well known [1-3], although the mechanisms of autoimmune diseases associated with primary immune deficiency are not fully described [3-5]. Autoimmune diseases are the most frequent in common variable immune deficiency (CVID) and selected IgA deficiency (IgAD) [6-14].

The diagnosis of CVID is based on a significantly reduced level of IgG and/or IgA and IgM, accompanied by impaired or absent production of specific antibodies. The disturbances in cellular immunity include a decrease of lymphocyte number, weak response to mitogens and antigens, and reduced production of cytokines. The common clinical symptoms of CVID are recurrent bacterial infections, mainly involving the respiratory tract, with a poor response to antibiotic therapy and prolonged course $[1,7,9]$. Chronic sinusitis and bronchiectasis are frequent in older children and adults after years of CVID independently of regular immunoglobulin substitution in a prophylactic dose [9].

The wide range of clinical features associated with CVID includes gastrointestinal symptoms. Depending on the studied group, gastrointestinal (GI) involvement was 
noted in $20 \%$ up to $60 \%$ of CVID patients [2, 9, 15, 16] It is believed that in children with CVID, the gastrointestinal symptoms lead to underweight and inhibition of growth followed by jejunal dysfunction and malabsorption. However, as the symptoms overlap, the decreased weight and inhibition of growth might be explained by frequent infections, chronic inflammation and different therapies used during infections. The occurrence of autoimmune diseases in CVID is very common, but the profile of autoimmune diseases differs between children and adults. Gastrointestinal diseases, e.g. celiac disease or Crohn's disease, are more often noted in CVID and IgAD patients diagnosed in childhood than in those diagnosed later in life. Moreover, the clinical symptoms and the histology of jejunum biopsy from children with CVID showed characteristic differences as compared to children without immune deficiency [17]. In histology, the lack of plasma cells is typical and most striking. The increase of number of intraepithelial lymphocytes (IEL), granulomas and crypt distortion are characteristic and often mimic other features, e.g. graft-versus host disease $[2,15]$. These differences have led to inflammatory GI diseases in CVID patients being named as "sprue-like", "celiac-like" and "Crohn's-like" [2]. In children with the diagnosis of CVID but preserved production of IgA (tested in the serum as representative for secretory IgA produced in the jejunum), the infiltrations and production of autoantibodies within infiltrates under the mucous membrane are similar to those seen in children without CVID, which stresses the role of secretory lgA for gastrointestinal tract function [2].

Patients with IgAD have an increased risk of organ-specific autoimmune disease, e.g. diabetes mellitus type 1 , autoimmune thyroiditis and celiac disease. The prevalence of celiac disease in IgAD patients is about 3\%, which represents a 10-16-fold increase as compared to the general population [2, 4]. The clinical symptoms are often unspecific, suggesting a late-onset, hidden or asymptomatic form of celiac disease. In children without immune deficiency, the immunological detection of celiac disease based on autoantibodies and IgA class antibodies in the serum is clinically valuable as a screening test. In CVID and IgAD patients, the results of tests based on IgA may be false negative and the results of tests based on IgG should be considered as valuable and clinically significant $[2,18,19]$.

The problem of antibody production in CVID is more complex, since we know that the regular intravenous substitution of IgG, even in prophylactic doses, might be suppressive for own production of immunoglobulin including specific antibodies [20]. However, this suppression seems to be weak and the situation of permanent stimulation of the immune system with present antigens or autoanti- gens may overcome this suppression. The common view that in CVID the production of specific antibodies is low or absent was based on assays of response to vaccines or bacterial antigens from infections presented to the immune system in single doses $[1,9]$. The prolonged exposure of immune-competent cells to antigens in unknown; long duration of the ongoing autoimmune process in subclinical forms is a possible explanation for autoantibody production by humoral immune deficiency patients.

In inflammatory diseases of the Gl, e.g. Crohn's disease or ulcerative colitis, the increase of mucous membrane permeability for bacterial and yeast antigens induces uncontrolled migration of various antigens through the membrane followed by contact with lymphoid tissues. In consequence, the process of inflammation begins and is continued supported by increasing infiltration of immuno-competent cells, local production of proinflammatory cytokines and induction of a humoral response $[2,15,21]$. The permanent stimulation is probably sufficient to stimulate the immune system for the production of different types of antibodies, including autoantibodies, even in CVID patients. It is the rationale for detection of antibodies in the serum of CVID patients. However, in these patients, there is a possibility that the antibodies may be seen not in all individuals despite the ongoing autoimmune process. In patients with low production of antibodies (below detection or border level) and subclinical course, autoimmune gastrointestinal diseases are undiagnosed or delayed in diagnosis until more specific symptoms occur and/or antibody levels increase.

In IgAD patients, the production of antibodies is possible only in IgG class and tests using IgG are preferred for the diagnosis of autoimmune disease. Moreover, there is no immunoglobulin substitution in these children, so the results in IgG class are valuable and clinically significant. The possibility of detection of antibodies helps to diagnose autoimmune diseases in IgAD patients reasonably early. The clinical significance of early diagnosis is obvious, especially when the therapy, e.g. in celiac disease, is a gluten-free diet (GFD) and may be introduced immediately. However, observations of CVID patients with celiac disease showed lack of effectiveness of GFD recognized as refractory celiac disease (RCD) with progression of malabsorption syndrome in individual patients [22]. It indicates that autoimmune and/or chronic inflammatory processes are different in immune deficiency patients and the standard therapy may fail.

\section{Aim}

The purpose of the study was to show the usefulness of serological tests for celiac disease and IBD as screening and diagnostic procedures in patients with IgAD and CVID. 
Table I. Characteristics of patients included in the study (group I and II)

\begin{tabular}{lcc} 
Clinical symptoms & $\begin{array}{c}\text { Group I } \\
\text { CVID; 43 patients }\end{array}$ & $\begin{array}{c}\text { Group II } \\
\text { IgAD; 63 patients }\end{array}$ \\
\hline Infections of respiratory tract & 25 & 40 \\
\hline Thrombocytopenia & 3 & 0 \\
\hline Leukopenia & 4 & 0 \\
\hline Celiac disease (diagnosed before study) & 1 & 2 \\
\hline Bronchitis asthma & 4 & 6 \\
\hline Allergy (atopic dermatitis, food allergy) & 2 & 8 \\
\hline Autism & 2 & 0 \\
\hline Cardiomyopathy (diagnosed at age 6 months) & 1 & 0 \\
\hline Lymphocytic infiltrating pneumonia (LIP) & 1 & 0 \\
\hline Diabetes type 1 & 0 & 1 \\
\hline Epilepsy & 0 & 1 \\
\hline Regular immunoglobulin substitution & $1-9$ years (mean - 3.64 years) & no
\end{tabular}

\section{Material and methods \\ Patients}

One hundred and six children (37 girls, 69 boys, aged 1-18 years) with the diagnosis of humoral immune deficiency were included in the study. Based on the diagnosis, the following groups were described:

Group I: 43 children (12 girls, 31 boys, mean age 9 years) -37 patients with CVID, 5 patients with hypogammaglobulinemia and 1 patient with lack of specific antibody synthesis. Patients from this group are on regular substitution of immunoglobulins (mean time 3 years, 2 months);

Group II: 63 patients (25 girls, 38 boys, mean age 7 years, 11 months) with the diagnosis of isolated IgA deficiency (IgAD).

\section{Infections and other diseases in children with IgA deficiency and common variable immune deficiency}

In group I (43 patients), more than half of patients showed recurrent respiratory tract infections despite regular substitution of immunoglobulins. Hematological disorders included chronic thrombocytopenia (3 patients) and leukopenia (4 patients). In one girl, celiac disease had been diagnosed before this study with improvement (no clinical symptom or antibody production) on GFD. Six patients demonstrated allergy (4 - bronchial asthma, 2 - allergic rhinitis). Two patients with CVID had been diagnosed with autism before the diagnosis of CVID was established; another patient is being monitored by a cardiologist because of cardiomyopathy diagnosed in infancy. In the course of CVID, in one girl, the diagnosis of lymphocytic infiltrating pneumonia (LIP) was established followed with steroid therapy. In group II, infections of the upper respiratory tract were noted in $63 \%$ of the patients. Two patients had been diagnosed with celiac disease, six with bronchial asthma and 8 with allergic rhinitis and food allergy before this study. One patient had been diagnosed with diabetes type 1, now treated with insulin. One boy is on regular therapy against epilepsy (Table I).

\section{Methods}

In all the patients, detection of antibodies typical for inflammatory bowel diseases (IBD), celiac disease (CD) and atrophic gastritis (AG) was performed qualitatively with indirect immunofluorescence (IIF) and quantitative ELISA techniques. Standard kits from Euroimmun (Lubeck, Germany) were used containing the following tissue slides: pancreas, jejunum, stomach, esophagus, neutrophils and drops of gliadin extract (FA 1380, FA 1919, FA 1911). Additionally, screening tests for systemic autoimmune disease antibodies were performed with IIF (FA 1800-2005-6). The slides were assayed with a fluorescence microscope (Bx60, Olympus, Japan) under 100x magnification by two independent investigators. Highly purified antigens of Saccharomyces cerevisiae cell membrane (EV 2841-9601), enzyme tissue transglutaminase (EA 1910-9610) and parietal cell antigens (EV 1361-9610) (Euroimmun) were used in ELISA technique. The control sera (positive and negative) were run in parallel to patients' sera.

Antibodies for celiac disease:

- anti-endomysial antibodies (EmA) with IIF. Positive results are seen as linear fluorescence of reticulin present in smooth muscles and jejunum villi slides in patients' serum diluted $1: 10$; 
- anti-gliadin antibodies - (AGA) with IIF. Positive results are seen as fluorescence of purified gliadin droplets in the serum diluted $1: 10$;

- anti-tissue transglutaminase enzyme (tTGA) with ELISA kit. The dilution of the serum $1: 100$, positive results absorbance above $20 \mathrm{RU} / \mathrm{ml}$ according to the standard curve in IgA and IgG class according to the manufacturer's instructions. All the above tests were performed in IgA class and IgG class for studied patients.

Antibodies for Crohn's disease:

- anti-Saccharomyces cerevisiae antibodies (ASCA) with ELISA. Positive results - absorbance above $20 \mathrm{RU} / \mathrm{ml}$ according to the standard curve in the serum diluted $1: 100$ in IgA and IgG class;

- anti-exocrine pancreatic cells and their products (Pab) with IIF. Positive results are seen as bright fluorescence of pancreatic cell cytoplasm and droplets of produced enzymes in the serum diluted $1: 10$.

Antibodies for ulcerative colitis:

- antibodies for neutrophil cytoplasm antigens (ANCA) with IIF seen on human neutrophil slides. Positive results are seen as granular fluorescence within cytoplasm (atypical ANCA - aANCA) or linear perinuclear pattern (pANCA) in the serum diluted $1: 10$;

- antibodies for goblet cells (Gab) with IIF. Positive results are seen as bright fluorescence of goblet cell cytoplasm (mucins are an antigen) in the serum diluted $1: 10$.

Antibodies for atrophic gastritis:

- antibodies for parietal cells (PCA) with ELISA. Absorbance higher than $20 \mathrm{RU} / \mathrm{ml}$ according to the standard curve is considered as positive in the serum diluted $1: 100$ [14].

Antibodies for systemic autoimmune disease (screening):

- for nuclear antigens (ANA), for smooth muscles (SMA), mitochondrial (AMA) and liver-kidney microsomal antigen (LKMS) with IIF in the serum diluted $1: 100$. The fluorescence of the patient's serum was compared to positive and negative controls.

Because of immune deficiency the limit of positive results for antibody presence in serum tested with ELISA was lowered to $10 \mathrm{RU} / \mathrm{ml}$ for CVID patients and routinely performed in IgG class for IgAD and CVID (without IgA) patients.

\section{Results}

\section{Frequency and type of antibodies \\ for autoimmune diseases of gastrointestinal tract}

In the analyzed group of 106 patients, antibodies typical for IBD were noted in 17 patients (16.0\%). The types of antibodies were as follows: for ASCA in IgG class - 11 patients, IgA and IgG class -2 patients and IgA class only - 1 patient, ANCA - 2 patients and Gab 1 patient.

Antibodies for celiac disease were noted in 16 patients (15.0\%) - AGA - 10 patients, tTG - 3 patients (in 2 cases together with EmA) and EmA (in IgG class) in 3 patients. PCA were seen in the sera of 5 patients (4.71\%) (Table II).

\section{The relation of antibodies to type of immune deficiency \\ Group I (43 patients)}

Antibodies for IBD were noted in 7 patients (16.27\%): ASCA in IgG class - 4 patients, ASCA in both IgG and IgA class - 2 patients, and ASCA in IgA class 1 patient. Antibodies typical for celiac disease were noted in 3 patients $(6.9 \%)$ - EmA in IgA class together with tTG and AGA in 2 patients, followed by clinical and histological diagnosis of celiac disease. Additionally, AGA in IgG class were seen in one patient's serum.

\section{Group II (63 patients)}

Antibodies for IBD were noted in 10 patients (15.87\%): ASCA in IgG class - 7 patients, ANCA - 2 patients, and Gab - 1 patient. Antibodies typical for celiac disease were found 13 patients (20.6\%) - AGA in 9 patients, tTG (in IgA and IgG class) in 1 patient (now under diagnostic procedure of celiac disease) and EmA in 3 patients.

The frequency and type of antibodies in relation to diagnosis are shown in Table II.

Clinical symptoms in patients with IgA deficiency and common variable immune deficiency and present antibodies

All the patients with present antibodies were examined for IBD and celiac disease according to standard procedures. In group I, clinical gastrointestinal tract symptoms were found in 3 patients, including two with newly diagnosed celiac disease. These symptoms were mild and unspecific, e.g. underweight and low gain of weight, feeling of discomfort after meals. In both celiac patients, the clinical course was different than in children without immune deficiency. The first patient with mild Gl symptoms without changes in jejunum histology (Marsh 0) improved on GFD. The second celiac patient demonstrated a high level of tTG antibodies for more than one year of observation despite restricted GFD.

Moreover, what seems to be interesting is the presence of PCA in children with IGAD and CVID without clinical and histological symptoms of an autoimmune process.

In both groups (IgAD and CVID), there were patients without antibodies, but with some mild gastrointestinal 
Table II. Occurrence of antibodies typical for gastrointestinal autoimmune diseases (celiac disease and IBD) in CVID and IgAD patients included in the study

\begin{tabular}{|c|c|c|c|c|c|}
\hline Antibodies for: & & $\begin{array}{c}\text { Group I } \\
\text { CVID }(n=43)\end{array}$ & $\begin{array}{c}\text { Group II } \\
\text { IgAD }(n=63)\end{array}$ & $\begin{array}{l}\text { All patients } \\
(n=106)\end{array}$ & Total \\
\hline \multirow[t]{5}{*}{ IBD } & ASCA (IgG) & 4 & 7 & 11 & \multirow{5}{*}{$17(16.0 \%)$} \\
\hline & ASCA $(\lg G+\operatorname{Ig} A)$ & 2 & 0 & 2 & \\
\hline & ASCA (IgA) & 1 & 0 & 1 & \\
\hline & ANCA & 0 & 2 & 2 & \\
\hline & Gab & 0 & 1 & 1 & \\
\hline \multirow[t]{3}{*}{ Celiac disease } & $t T G+E m A+A G A$ & 2 & 1 & 3 & \multirow{3}{*}{$16(15.09 \%)$} \\
\hline & EmA & 0 & 3 & 3 & \\
\hline & AGA & 1 & 9 & 10 & \\
\hline Atrophic gastritis & PCA & 2 & 3 & 5 & $5(4.71 \%)$ \\
\hline \multirow{2}{*}{$\begin{array}{l}\text { Systemic } \\
\text { autoimmune diseases }\end{array}$} & ANA & 5 & 5 & 10 & $10(9.43 \%)$ \\
\hline & SMA & 3 & 0 & 3 & $3(3.44 \%)$ \\
\hline Total & & $20(46.5 \%)$ & 31 (47.61\%) & 51 (48.11\%) & \\
\hline
\end{tabular}

tract symptoms: 5 patients in group I, and 4 patients in group II. These children are being monitored very carefully.

\section{Autoantibodies for systemic autoimmune diseases}

In group I, ANA were detected in 5 patients, and SMA in 3 patients. In group II, ANA were found in 5 patients (Table II). These antibodies were considered as markers of possible autoimmune phenomena only. A retest is suggested every 6 months if clinical symptoms do not occur.

\section{Conclusions}

In children with CVID and IgAD, detection of autoantibodies every 4-6 months is required to select patients at high risk of autoimmune diseases, especially involving the gastrointestinal tract. Autoantibodies for autoimmune diseases were noted in about half of children with CVID and IgAD, without any typical clinical symptoms of the diseases in the majority of them. In children without clinical symptoms, but with antibodies present for 1 year or more, diagnostic procedures including gastroscopy, colonoscopy and biopsy are indicated to diagnose IBD or celiac disease in a subclinical form. The detailed analysis of jejunum histology including atypical features is suggested.

\section{Discussion}

The precise and sensitive methods of detection of various antibodies have shown the possibility of sero- logic screening and supporting the diagnosis of autoimmune disease in patients with immune deficiency, e.g. CVID and IgAD. Moreover, regular substitution of IgG in CVID patients is not suppressive enough to inhibit the autoantibody production below the level of detection in the serum.

In our study, the antibodies were noted in almost half of the patients with the diagnosis of IgAD and CVID. In CVID, antibodies for IBD and celiac disease were present in $23 \%$ of patients with the presence of ASCA, which suggests the possibility of development of Crohn's disease. In IgAD, antibodies for celiac disease were noted in $20 \%$ of the patients, which supported observations of increased risk of this disease in IgAD. Moreover, due to the possibility of ongoing celiac disease in latent, asymptomatic form, clinical and laboratory diagnostic procedures are indicated as the subsequent steps without any undue time loss [19, 23]. All these patients were without typical clinical symptoms suggestive of a particular inflammatory disease. Only two patients showed mild gastrointestinal symptoms and celiac disease was diagnosed by biopsy in one of them; in the second case, histology showed no typical changes (grade 0 according to the Marsh scale). The GFD was introduced in both cases with improvement of patients (e.g. increase of weight). The remaining patients positive for IBD or celiac disease antibodies are being monitored (the antibody panel is tested every 6 months). Clinical follow-up is careful; all symptoms, even mild, are noted and analyzed in detail.

In children with isolated IgAD, celiac disease is the most common as an autoimmune association. The de- 
tection of antibodies in IgG class is used for screening, initial diagnosis and monitoring during GFD. In these patients, antibodies in class IgG are clinically significant as compared to antibodies in IgA class in patients without IgAD [2, 23]. In adults with IgAD, systemic lupus erythematosus (SLE) is frequently observed and antinuclear antibodies are used for diagnosis, similarly as in patients with normal IgA production. In children, ANA are considered a signal of a possible process and monitored only, as SLE is rare in children with IgAD [12, 13]. The presence of ANA is an indication for prolonged observation only without the possibility of predicting the development of clinical symptoms of disease [14].

The diagnosis of autoimmune diseases in patients with immune deficiency is problematic for two reasons: one of them is the low production of antibodies in case of a decreased number of $B$ lymphocytes and the presence of antibodies without clinical symptoms of disease; and the second is the possible suppressive effect of regular substitution of IgG (e.g. in CVID). These two phenomena may lead to low levels of autoantibodies detected in the serum. Considering these two aspects of antibody detection in patients with humoral immune deficiency, modification of interpretation of the antibody detection results seems to be a reasonable solution. The decrease of the lower limit for positive results is the basic modification, so the antibodies present in lower titer are accepted as valuable and clinically significant. However, in single observation the level of ANA before and after intravenous infusions of immunoglobulins ( $0.4 \mathrm{~g} / \mathrm{kg}$ b.w. dose) was unchanged, which suggested that the induced production of autoantibodies is strong enough to be preserved despite immunoglobulin substitution. It may be speculated that this suppressive effect of immunoglobulin in a low dose prevents the occurrence of autoantibody production but is too weak when this process was induced. The autoantibodies are involved in reactions with antigens (autoantigens) present in the tissue of the target organ for this autoimmune process. In the serum, the circulating antibodies are seen when the production is higher than uptake by the tissue. In the case of low production and an ongoing process of reaction with antigens, the level of antibodies in the serum is below the detection level. This explains cases when an autoimmune process is seen e.g. in the histology of the affected tissue/organ, but the circulating antibodies are absent in the serum. In children with immune deficiency, this possibility is important because the lack of antibodies does not exclude the autoimmune process leading to tissue damage. The opposite situation is noted when the antibodies are present without any clinical symptoms, in which case they may be considered as markers of an ongoing autoimmune process in subclinical form. These patients are under long-term observation with regular checking of antibodies and occurrence of clinical symptoms. The presence of antibodies is often observed as a phenomenon preceding the autoimmune process and onset of clinical symptoms. Moreover, in these patients, the differential diagnostic procedures include a wide range of tests and methods, e.g. for bacterial overgrowth in the jejunum, bacterial characteristics of the stool, presence of parasites (e.g. Giardia lamblia), and parameters suggesting malabsorption (e.g. iron level, aphthae, osteopenia), to prove or exclude the autoimmune disease.

The course, symptoms and histology of autoimmune diseases in immune deficient patients are different as compared to the characteristics of similar diseases observed in patients without immune deficiency. In adult patients with CVID, autoimmune gastritis, pernicious anemia, Crohn's disease and ulcerative colitis are more frequent than in children. The differences of autoimmune diseases between healthy and immune deficient patients are seen in both aspects - clinical and laboratory. Patients with immune deficiency often present scarce, unspecific clinical symptoms that overlap with the effects of frequent infections and chronic inflammatory complications. In the histology of jejunum biopsy material, changed proportions of T lymphocyte subpopulations (TCR $\alpha / \beta$ : TCR $\gamma / \delta$ ) and an increased number of Tregs are noted [15]. Intraepithelial lymphocytosis, lymphoid aggregates, granulomas, crypt distortion and lack of plasma cells are characteristic. Moreover, in inflammatory bowel-like disease, the histological pattern of biopsy in CVID patients often mimics graft-versushost disease, lymphoid hyperplasia and villous atrophy that resembles celiac disease [13, 22].

The problem of low or lack of specific antibody secretion in CVID is not as common as would be expected. The antibodies in IgA class are detected in a reasonable number of patients and the clinical significance of these antibodies is high. The problem is in patients without circulating antibodies, but in the case of clinical symptoms, biopsy is indicated because of its basic and high diagnostic value.

\section{References}

1. Agarwal S, Cunningham-Rundles C. Autoimmunity in common variable immune deficiency. Curr Allergy Asthma Rep 2009; 9: 347-52.

2. Agarwal S, Mayer L. Pathogenesis and treatment of gastrointestinal disease in antibody deficiency syndromes. J Allergy Clin Immunol 2009; 1245: 658-64.

3. Arason GJ, Jorgensen GH, Ludviksson BR. Primary immune deficiency and autoimmunity: lessons from human diseases. Scand J Immunol 2010; 71: 317-28. 
4. Arkwright PD, Abinun M, Cant AJ. Autoimmunity in primary immune deficiency diseases. Blood 2002; 99: 2694-702.

5. Fairweather D, Kaya Z, Shellam GR, et al. From infection to autoimmunity. J Autoimmunity 2001; 16: 175-86.

6. Boileau J, Mouillot G, Gerard L, et al. Autoimmunity in common variable immune deficiency: correlation with lymphocyte phenotype in the French DEFI study. J Autoimmunity 2010; 35: 1-8.

7. Brandt D, Gershwin ME. Common variable deficiency and au toimmunity. Autoimm Rev 2006; 5: 465-70.

8. Cunningham-Rundles C. Hematologic complications of primary immune deficiencies. Blood Rev 2002; 16: 61-4.

9. Cunnigham-Rundles C, Bodian C. Common variable immune deficiency: clinical and immunological features of 248 patients Clin Immunol 1999; 92: 34-48.

10. Knight AK, Cunningham-Rundles C. Inflammatory and autoimmune complications of common variable immune deficiency. Autoimm Rev 2006; 5: 156-9.

11. Mackay IR, Leskovsek NV, Rose NR. The old couple: a fresh look at autoimmunity and immune deficiency. J Autoimmunity 2010; 35: 199-205.

12. Sarmiento E, Mora R, Rodriguez-Mahou M, et al. Autoimmune disease in primary antibody deficiencies. J Allergol Immunopathol 2005; 33: 69-73.

13. Aytekin C, Tuygun N, Gokce S, et al. Selective IgA deficiency:clinical and laboratory features of 118 children. J Clin Immunol 2012; 32: 961-6.

14. Gulez N, Karaca NE, Aksu G, Kutukculer N. Increased percentage of autoantibodies in immunoglobulin-A-deficient children do not correlate with clinical manifestations. Autoimmunity 2009; 42: 74-9.

15. Daniels JA, Lederman HM, Maitra A, Montgomery EA. Gastrointestinal tract pathology in patients with common variable immune deficiency (CVID). Am J Surg Pathol 2007; 31: 1800-12.

16. Tozzoli R, Kodermaz G, Perosa AR, et al. Autoantibodies to parietal cells as predictors of atrophic body gastritis. Autoimm Rev 2010; 10: 80-3.

17. Borrelli M, Maglio M, Agnese M, et al. High density of intraepithelial gamma/delta lymphocytes and deposits of immunoglobulin (Ig)M anti tissue transglutaminase antibodies in the jejunum of celiac patients with IgA deficiency. Clin Exp Immunol 2009; 160: 199-206.

18. Villalta D, Tonutti E, Prause C, et al. IgG antibodies against deamidated gliadin peptides for diagnosis of celiac disease in patients with IgA deficiency. Clin Chem 2010; 56: 464-8.

19. Iwańczak, F, Iwańczak P. New guidelines for diagnosis and treatment of coeliac disease in children and adolescents. Prz Gastroenterol 2012; 7: 185-91.

20. Nimmerjahn F, Ravetch JV. Anti-inflammatory actions of intravenous immunoglobulin. Annu Rev Immunol 2008; 26: 513-33.

21. Kaser A, Zeissig S, Blumberg RS. Inflammatory bowel disease. Annu Rev Immunol 2010; 28: 573-621.

22. Walker MM, Murray JA. An update in the diagnosis of coeliac disease. Histopathology 2010; 30: 203-8.

23. Pituch-Noworolska A, Zwonarz K. Celiac and inflammatory bowel diseases in children with primary humoral immunodeficiency. In: Autoimmune diseases - contributing factors, sopecific cases of autoimmune diseases and stem cells and other therapies. Chan J (ed.). Intech 2012; 151-72.
Received: 7.03.2013

Accepted: 3.06 .2013 